Technical Report

22 December 2007

\title{
Tight frames generated by finite nonabelian groups
}

\author{
Richard Vale and Shayne Waldron
}

\author{
Department of Mathematics, University of Glasgow, Glasgow G12 8QQ, United Kingdom \\ e-mail: rv@maths.gla.ac.uk \\ Department of Mathematics, University of Auckland, Private Bag 92019, Auckland, New Zealand \\ e-mail: waldron@math.auckland.ac.nz
}

\begin{abstract}
Let $\mathcal{H}$ be a Hilbert space of finite dimension $d$, such as the finite signals $\ell_{2}(d)$ or a space of multivariate orthogonal polynomials, and $n \geq d$. There is a finite number of tight frames of $n$ vectors for $\mathcal{H}$ which can be obtained as the orbit of a single vector under the unitary action of an abelian group $G$ (of symmetries of the frame). Each of these so called harmonic frames or geometrically uniform frames can be obtained from the character table of $G$ in a simple way. These frames are used in signal processing and information theory.

For a nonabelian group $G$ there are in general uncountably many inequivalent tight frames of $n$ vectors for $\mathcal{H}$ which can be obtained as such a $G$-orbit. However, by adding an additional natural symmetry condition (which automatically holds if $G$ is abelian), we obtain a finite class of such frames which can be constructed from the character table of $G$ in a similar fashion to the harmonic frames. This is done by identifying each $G$-orbit with an element of the group algebra $\mathbb{C} G$ (via its Gramian), imposing the condition in the group algebra, and then describing the corresponding class of tight frames.
\end{abstract}

Key Words: signal processing, information theory, finite nonabelian groups, representation theory, group matrices, tight frames, harmonic frames, geometrically uniform frames, Gramian matrix, central tight $G$-frames

AMS (MOS) Subject Classifications: primary 20C15, 42C15, 41A63, 41A65 secondary 65T60, 94A11, 94A12, 94A15 


\section{Introduction}

A tight frame for a finite dimensional Hilbert space $\mathcal{H}$ which is the orbit of a single vector under the unitary action of a finite group $G$ will be called a tight $G$-frame. For $G$ an abelian group, these frames are known in the literature as harmonic frames or geometrically uniform frames. They are used extensively in signal processing and information theory, see, e.g., [CK07], [EF01] and [BE03].

It was proved in [VW05:Th.5.4] that if $G$ is abelian then each tight $G$-frame is unitarily equivalent to a tight frame obtained by deleting rows of the character table of $G$. For example, if $G$ is the cyclic group of order 3 , then its character table is

$$
\left[\begin{array}{ccc}
1 & 1 & 1 \\
1 & \omega & \omega^{2} \\
1 & \omega^{2} & \omega
\end{array}\right], \quad \omega:=e^{\frac{2 \pi i}{3}}
$$

By deleting the first row we obtain 3 vectors $\phi_{0}, \phi_{1}, \phi_{2}$ which form a tight frame for $\mathbb{C}^{2}$

$$
\phi_{0}=\left[\begin{array}{l}
1 \\
1
\end{array}\right], \phi_{1}=\left[\begin{array}{c}
\omega \\
\omega^{2}
\end{array}\right], \phi_{2}=\left[\begin{array}{c}
\omega^{2} \\
\omega
\end{array}\right], \quad \phi_{j}=\left(\begin{array}{cc}
\omega & 0 \\
0 & \omega^{2}
\end{array}\right)^{j} \phi_{0} .
$$

This is unitarily equivalent to the tight frame of 3 equally spaced unit vectors in $\mathbb{R}^{2}$.

The purpose of this paper is to generalise the above result to nonabelian groups. By way of motivation, consider the smallest nonabelian group $G=D_{3} \approx S_{3}$, the dihedral group of order 6 , acting on $\mathbb{R}^{2}$ as a group of unitary transformations generated by the elements $a$ of rotation through $2 \pi / 3$, and $b$ of reflection in the $x$-axis. The $G$-orbit of each of the vectors

$$
v_{\theta}:=\left[\begin{array}{c}
\cos \theta \\
\sin \theta
\end{array}\right], \quad 0 \leq \theta \leq \frac{\pi}{6},
$$

is a tight $G$-frame for $\mathbb{R}^{2}$ (and $\mathbb{C}^{2}$ ). Since unitary maps preserve angles, none of these tight $G$-frames is a unitary image of another.
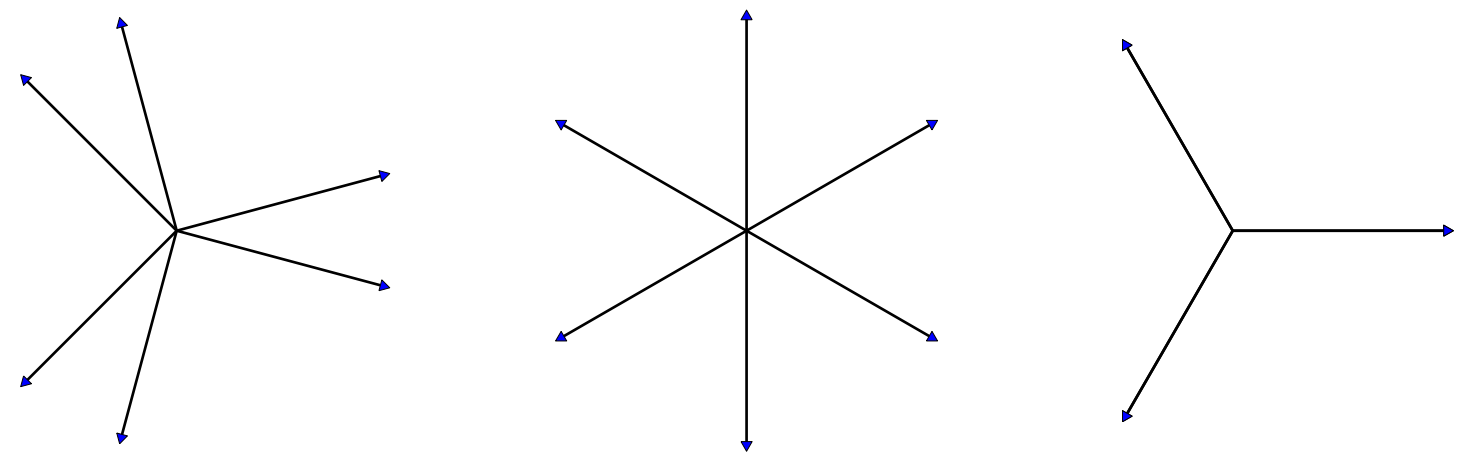

Fig 1. The tight $D_{3}$-frames obtained from $v_{\theta}$, for $\theta=\frac{\pi}{12}, \theta=\frac{\pi}{6}$ and $\theta=0$.

This example shows that for a finite nonabelian group $G$ the class of all inequivalent tight $G$-frames $\Phi=(g v)_{g \in G}$ may be uncountable, whereas for $G$ abelian it is finite. 
To narrow down this class, we impose an additional symmetry condition, which can be expressed as

$$
\langle g \phi, h \phi\rangle=\langle g \psi, h \psi\rangle, \quad \forall g, h \in G, \forall \phi, \psi \in \Phi .
$$

This yields a finite subset of the nicest such frames, which reduces to the harmonic frames when $G$ is abelian. It is hoped these frames will be useful in applications such as signal processing, along the lines of [BK00] and [AMS05]. Indeed, these frames already appear (for infinite groups $G$ ) as the Weyl-Heisenberg frames for subspaces of $L_{2}(\mathbb{R})$ which have a unique Weyl-Heisenberg dual in the given subspace [GH04 $]$, and the generators satisfy the Balian-Low uncertainty principle [GH04 2 .

The rest of the paper is set out as follows. Next we give the basic theory of frames for an index set without structure that we require. We then consider the case when the frame vectors are indexed by and can be obtained from the elements of a finite group $G$, the so called $G$-frames. We then show that the (normalised) tight $G$-frames can be identified, via their Gramian, with elements of the group algebra $\mathbb{C} G$. Finally, we obtain our class of nice tight $G$-frames, the so called central tight $G$-frames, by identifying them as elements of the centre of $\mathbb{C} G$. We give a complete structure theorem for these frames in terms of the characters of $G$, and a number of illustrative examples.

\section{The basics of frame theory}

The following results are known (see [HL00] and [VW05]). Since their statement in terms the Gramian given here is new, we provide proofs for completeness.

Let $\mathcal{H}$ be a Hilbert space of finite dimension $d$. A finite sequence of $n \geq d$ vectors $\left(\phi_{j}\right)_{j \in J}$ in $\mathcal{H}$ is a frame for $\mathcal{H}$ if it spans $\mathcal{H}$, and is a tight frame for $\mathcal{H}$ if there is a $c>0$ with

$$
f=c \sum_{j \in J}\left\langle f, \phi_{j}\right\rangle \phi_{j}, \quad \forall f \in \mathcal{H} .
$$

We consider tight frames $\left(\phi_{j}\right)_{j \in J}$ and $\left(\psi_{j}\right)_{j \in J}$ to be equivalent if there exists a unitary transformation $U$, and a $c>0$ for which

$$
\phi_{j}=c U \psi_{j}, \quad \forall j \in J .
$$

Any two such frames will be said to be (unitarily) equivalent, and we will talk of uniqueness of a frame up to this (unitary) equivalence.

We say a tight frame is normalised if it has been scaled so that $c=1$ in (2.1), i.e.,

$$
f=\sum_{j \in J}\left\langle f, \phi_{j}\right\rangle \phi_{j}, \quad \forall f \in \mathcal{H}
$$

Such frames are also known as Parseval tight frames. If $\left(\phi_{j}\right)_{j \in J}$ is a normalised tight frame with vectors of equal length, then taking the trace of the linear maps defined by (2.3) gives

$$
\operatorname{dim}(\mathcal{H})=\sum_{j \in J}\left\|\phi_{j}\right\|^{2} \Longrightarrow\left\|\phi_{j}\right\|=\sqrt{\frac{\operatorname{dim}(\mathcal{H})}{|J|}}, \quad \forall j \in J
$$


Definition. Let $\Phi=\left(\phi_{j}\right)_{j \in J}$ and $\Psi=\left(\psi_{j}\right)_{j \in J}$ be frames for finite dimensional Hilbert spaces $\mathcal{H}_{1}$ and $\mathcal{H}_{2}$. Their direct sum is defined to be

$$
\Phi \oplus \Psi:=\left(\phi_{j}+\psi_{j}\right)_{j \in J} \subset \mathcal{H}_{1} \oplus \mathcal{H}_{2}
$$

In general $\Phi \oplus \Psi$ is not a frame for the direct sum $\mathcal{H}_{1} \oplus \mathcal{H}_{2}$. However, a simple calculation shows that if $\Phi$ and $\Psi$ are normalised tight frames, then $\Phi \oplus \Psi$ is a normalised tight frame for $\mathcal{H}_{1} \oplus \mathcal{H}_{2}$ provided

$$
\sum_{j \in J}\left\langle f, \phi_{j}\right\rangle \psi_{j}=0, \quad \forall f \in \mathcal{H}_{1}, \quad \sum_{j \in J}\left\langle g, \psi_{j}\right\rangle \phi_{j}=0, \quad \forall g \in \mathcal{H}_{2}
$$

Definition. The Gramian of a frame $\Phi=\left(\phi_{j}\right)_{j \in J}$ with $|J|=n$ is the $n \times n$ matrix

$$
\operatorname{Gram}(\Phi):=\left[\left\langle\phi_{i}, \phi_{j}\right\rangle\right]_{i, j \in J}
$$

We now show that the possible Gramian matrices are precisely the orthogonal projections.

Theorem 2.5. An $n \times n$ matrix $P=\left[p_{i j}\right]_{i, j \in J}$ is the Gramian of a normalised tight frame $\left(\phi_{j}\right)_{j \in J}$ for the space $\mathcal{H}=\operatorname{span}\left\{\phi_{j}\right\}_{j \in J}$ if and only if it is an orthogonal projection matrix, i.e., $P=P^{*}=P^{2}$. Moreover, the dimension of the Hilbert space $\mathcal{H}$ for this frame is

$$
d=\operatorname{dim}(\mathcal{H})=\operatorname{rank}(P)=\operatorname{trace}(P)=\sum_{j \in J}\left\|\phi_{j}\right\|^{2}
$$

Proof: Let $\Phi=\left(\phi_{j}\right)_{j \in J}$ be a normalised tight frame, and $P=\operatorname{Gram}(\Phi)$. Take $f=\phi_{i}$ in (2.3) to get $\phi_{i}=\sum_{j \in J}\left\langle\phi_{i}, \phi_{j}\right\rangle \phi_{j}$, and take the inner product with $\phi_{k}$ to obtain

$$
\left\langle\phi_{i}, \phi_{k}\right\rangle=\sum_{j \in J}\left\langle\phi_{i}, \phi_{j}\right\rangle\left\langle\phi_{j}, \phi_{k}\right\rangle \quad \Longleftrightarrow \quad P_{i k}=\sum_{j \in J} P_{i j} P_{j k} \quad \Longleftrightarrow \quad P=P^{2} .
$$

Further $P$ is Hermitian since $\overline{P_{i j}}=\overline{\left\langle\phi_{i}, \phi_{j}\right\rangle}=\left\langle\phi_{j}, \phi_{i}\right\rangle=P_{j i}$, and hence is a projection.

Conversely, suppose that $P$ is an $n \times n$ matrix such that $P=P^{*}=P^{2}$. Let $\left\{e_{1}, \ldots, e_{n}\right\}$ be the standard basis of $\mathbb{C}^{n}$, so that the columns of $P$ are $\phi_{j}:=P e_{j}, j=1, \ldots n$. Fix $f \in \mathcal{H}:=\operatorname{span}\left\{\phi_{j}\right\}_{j=1}^{n} \subset \mathbb{C}^{n}$. Then $f=P f$, so that

$$
f=\sum_{j=1}^{n}\left\langle P f, e_{j}\right\rangle e_{j}=\sum_{j=1}^{n}\left\langle f, P e_{j}\right\rangle e_{j}=P \sum_{j=1}^{n}\left\langle f, P e_{j}\right\rangle e_{j}=\sum_{j=1}^{n}\left\langle f, P e_{j}\right\rangle P e_{j}=\sum_{j=1}^{n}\left\langle f, \phi_{j}\right\rangle \phi_{j},
$$

i.e., $\left(\phi_{j}\right)_{j=1}^{n}$ is a normalised tight frame for $\mathcal{H}$.

Finally, taking the trace of $P$ gives

$$
d=\operatorname{dim}(\mathcal{H})=\operatorname{rank}(P)=\operatorname{trace}(P)=\sum_{j \in J}\left\|\phi_{j}\right\|^{2} .
$$


It follows from Theorem 2.5 that normalised tight frames are unitarily equivalent if and only if their Gramians are equal (cf [VW05:Th.2.9]). In light of this, it should be possible to determine all properties of a normalised tight frame from its Gramian. We utilise this general principle in the remaining sections.

Corollary 2.7. If $\Phi=\left(\phi_{j}\right)_{j \in J}$ and $\Psi=\left(\psi_{j}\right)_{j \in J}$ are normalised tight frames, then

$$
\Phi \oplus \Psi:=\left(\phi_{j}+\psi_{j}\right)_{j \in J}
$$

is a normalised tight frame if and only if the product of the Gramians of $\Phi$ and $\Psi$ is zero. The Gramian of this frame is given by

$$
\operatorname{Gram}(\Phi \oplus \Psi)=\operatorname{Gram}(\Phi)+\operatorname{Gram}(\Psi) .
$$

Proof: The Gramian of $\Phi \oplus \Psi$ is the Hermitian matrix

$\operatorname{Gram}(\Phi \oplus \Psi)=\left[\left\langle\phi_{i}+\psi_{i}, \phi_{j}+\psi_{j}\right\rangle\right]_{i, j \in J}=\left[\left\langle\phi_{i}, \phi_{j}\right\rangle+\left\langle\psi_{i}, \psi_{j}\right\rangle\right]_{i, j \in J}=\operatorname{Gram}(\Phi)+\operatorname{Gram}(\Psi)$.

Since $P:=\operatorname{Gram}(\Phi)$ and $Q:=\operatorname{Gram}(\Psi)$ are orthogonal projections, this is an orthogonal projection if and only if

$$
(P+Q)^{2}=P^{2}+P Q+Q P+Q^{2}=P+P Q+Q P+Q=P+Q
$$

i.e., $P Q+Q P=0$. It is easy to check that $P Q+Q P=0$ if and only if $P Q=0$.

Example. Suppose that $\Phi$ is a normalised tight frame of $n$ vectors for a $d$-dimensional space, and $P=\operatorname{Gram}(\Phi)$. Then $I_{n}-P$ is a projection of rank $n-d$, which is therefore the Gramian of a normalised tight frame of $n$ vectors for a space of $n-d$ dimensions. This frame is unique (up to unitary equivalence) and we call it the complementary frame (cf [HL00] and [RW02]), and denote it by $\Phi^{c}$. The direct sum $\Phi \oplus \Phi^{c}$ has Gramian the identity, and hence is an orthonormal basis.

\section{Representations and $G$-frames}

Let $G$ be an abstract finite group. A representation of $G$ is a finite dimensional complex vector space $V$ together with a linear action of $G$ on $V$, or equivalently a group homomorphism $\rho_{V}: G \rightarrow G L(V)$. Two representations $\left(V, \rho_{V}\right)$ and $\left(W, \rho_{W}\right)$ are said to be equivalent if there is an invertible linear map $T: V \rightarrow W$ such that

$$
\rho_{W}(g)=T \rho_{V}(g) T^{-1}, \quad \forall g \in G .
$$

Any representation of $G$ is equivalent to a representation in which $V$ is a Hilbert space and all the $\rho_{V}(g)$ are unitary matrices. Hence in this paper we define a representation to be a finite dimensional Hilbert space $\mathcal{H}$ together with a group homomorphism $\rho: G \rightarrow U(\mathcal{H})$. We will often write the unitary group action induced by the representation as

$$
g v:=\rho(g)(v), \quad g \in G, v \in \mathcal{H} .
$$

The following definition was also given independently by [H07]. 
Definition. Let $G$ be a finite group. We say that a frame $\left(\phi_{g}\right)_{g \in G}$ for $\mathcal{H}$ is a $G$-frame if there exists a representation $\rho: G \rightarrow \mathcal{H}$ such that

$$
g \phi_{h}:=\rho(g) \phi_{h}=\phi_{g h}, \quad \forall g, h \in G .
$$

Remark. By this definition, the sequence $\Phi=\left(\phi_{g}\right)_{g \in G}=\left(g \phi_{1}\right)_{g \in G}$ consists of the $N$ elements of the $G$-orbit of $\phi_{1}$ (or any $\phi_{g}$ ) each repeated $|G| / N$ times. This "repetition" has certain technical advantages, e.g., for taking sums and complements of tight $G$-frames. If one is interested only in the frame obtained by taking the set of vectors making up a $G$-frame $\Phi$, then this can often be obtained as an $H$-frame of distinct vectors, where $H$ is a suitable subgroup or quotient of $G$. For example, the set of three equally spaced vectors in Figure 1, obtained by taking the $S_{3}$-orbit of $v_{0}$ is not a tight $S_{3}$-frame, but it is a $C_{3}$-frame (for $C_{3}$ the cyclic group of order three generated by the rotation $a$ ).

Example. Many results for frames indexed by a set $J$ carry over in a natural way to $G$-frames. For example, the complement of a $G$-frame is a $G$-frame, and the direct sum of normalised tight $G$-frames is a normalised tight $G$-frame.

We will freely use a number of basic definitions and results from representation theory. These can all be found in a standard textbook such as [L77], [JL93] or [I06].

\section{The identification of $G$-frames with the group algebra}

If $\Phi=\left(\phi_{g}\right)_{g \in G}$ is a $G$-frame, then the entries of its Gramian $\left[\left\langle\phi_{g}, \phi_{h}\right\rangle\right]_{g, h \in G}$ have the special form

$$
\left\langle\phi_{g}, \phi_{h}\right\rangle=\left\langle g \phi_{1}, h \phi_{1}\right\rangle=\left\langle\phi_{1}, g^{-1} h \phi_{1}\right\rangle .
$$

This is an example of what is called a group matrix or $G$-matrix.

Definition. Let $G$ be a finite group. Then a matrix $A=\left[a_{g h}\right]_{g, h \in G}$ is called a $G$-matrix if there exists a function $\nu: G \rightarrow \mathbb{C}$ such that

$$
a_{g h}=\nu\left(g^{-1} h\right), \quad \forall g, h \in G
$$

Remark. In [VW05] we used the term $G$-circulant for a $G$-matrix, since for $G$ a cyclic group, a $G$-matrix is a circulant matrix. We have since learned that we are part of a revival of interest in group matrices, see, e.g., [BR04], [J07] or [D79] (for $G$ the cyclic group).

It is also natural to think of an $n \times n$ matrix $A$ as a $G$-matrix for $G$ of order $n$ if there is an indexing of its entries by the elements of $G$ which yields a $G$-matrix (our orginal definition of being $G$-circulant), but this is less convenient for describing the algebraic properties of group matrices.

Example. Let $G=D_{3} \approx S_{3}$ be the dihedral group of order 6, i.e.,

$$
G=D_{3}=\left\langle a, b: a^{3}=1, b^{2}=1, b^{-1} a b=a^{-1}\right\rangle,
$$


and order its elements $1, a, a^{2}, b, a b, a^{2} b$. Then each $G$-matrix has the form

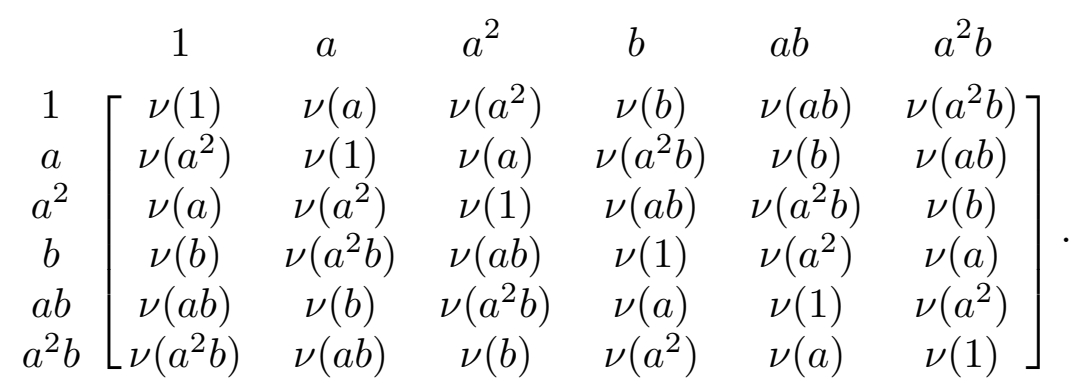

We now show that $\Phi$ is a $G$-frame if and only if its Gramian is a $G$-matrix.

Theorem 4.4. Let $G$ be a finite group. Then $\Phi=\left(\phi_{g}\right)_{g \in G}$ is a $G$-frame (for its span) if and only if its Gramian is a $G$-matrix.

Proof: If $\Phi$ is a $G$-frame, then (4.1) implies that its Gramian is a $G$-matrix.

Conversely, suppose that the Gramian of a frame $\Phi$ for $\mathcal{H}$ is a $G$-matrix. The frame operator $S: \mathcal{H} \rightarrow \mathcal{H}$ is the positive definite operator given by

$$
S(f):=\sum_{g \in G}\left\langle f, \phi_{g}\right\rangle \phi_{g}, \quad \forall f \in \mathcal{H} .
$$

With $\tilde{\phi}_{g}:=S^{-1} \phi_{g}$, we have the corresponding frame decomposition

$$
f=\sum_{g \in G}\left\langle f, \tilde{\phi}_{g}\right\rangle \phi_{g}, \quad \forall f \in \mathcal{H} .
$$

For $g \in G$, define $U_{g}: \mathcal{H} \rightarrow \mathcal{H}$ by

$$
U_{g}(f):=\sum_{h_{1} \in G}\left\langle f, \tilde{\phi}_{h_{1}}\right\rangle \phi_{g h_{1}}, \quad \forall f \in \mathcal{H} .
$$

Since the Gramian of $\Phi$ is a $G$-matrix, we have

$$
\left\langle\phi_{g h_{1}}, \phi_{g h_{2}}\right\rangle=\nu\left(\left(g h_{1}\right)^{-1} g h_{2}\right)=\nu\left(h_{1}^{-1} h_{2}\right)=\left\langle\phi_{h_{1}}, \phi_{h_{2}}\right\rangle .
$$

It follows from (4.5) and (4.6) that $U_{g}$ is unitary by the calculation

$$
\begin{aligned}
\left\langle U_{g}\left(f_{1}\right), U_{g}\left(f_{2}\right)\right\rangle & =\left\langle\sum_{h_{1} \in G}\left\langle f_{1}, \tilde{\phi}_{h_{1}}\right\rangle \phi_{g h_{1}}, \sum_{h_{2} \in G}\left\langle f_{2}, \tilde{\phi}_{h_{2}}\right\rangle \phi_{g h_{2}}\right\rangle \\
& =\sum_{h_{1} \in G} \sum_{h_{2} \in G}\left\langle f_{1}, \tilde{\phi}_{h_{1}}\right\rangle \overline{\left\langle f_{2}, \tilde{\phi}_{h_{2}}\right\rangle}\left\langle\phi_{g h_{1}}, \phi_{g h_{2}}\right\rangle \\
& =\sum_{h_{1} \in G} \sum_{h_{2} \in G}\left\langle f_{1}, \tilde{\phi}_{h_{1}}\right\rangle \overline{\left\langle f_{2}, \tilde{\phi}_{h_{2}}\right\rangle}\left\langle\phi_{h_{1}}, \phi_{h_{2}}\right\rangle \\
& =\left\langle\sum_{h_{1} \in G}\left\langle f_{1}, \tilde{\phi}_{h_{1}}\right\rangle \phi_{h_{1}}, \sum_{h_{2} \in G}\left\langle f_{2}, \tilde{\phi}_{h_{2}}\right\rangle \phi_{h_{2}}\right\rangle=\left\langle f_{1}, f_{2}\right\rangle .
\end{aligned}
$$


Similarly, we have

$$
U_{g} \phi_{h}=\sum_{h_{1} \in G}\left\langle\phi_{h}, \tilde{\phi}_{h_{1}}\right\rangle \phi_{g h_{1}}=\sum_{h_{1} \in G}\left\langle\phi_{g h}, \tilde{\phi}_{g h_{1}}\right\rangle \phi_{g h_{1}}=\phi_{g h}
$$

This implies $\rho: g \mapsto U_{g}$ is a group homomorphism since

$$
U_{g_{1} g_{2}} \phi_{h}=\phi_{g_{1} g_{2} h}=U_{g_{1}} \phi_{g_{2} h}=U_{g_{1}} U_{g_{2}} \phi_{h}, \quad \mathcal{H}=\operatorname{span}\left\{\phi_{h}\right\}_{h \in G} .
$$

Thus $\rho$ is a representation of $G$ with $\rho(g) \phi_{h}=\phi_{g h}$, i.e., $\Phi$ is a $G$-frame for $\mathcal{H}$.

Combining Theorems 2.5 and 4.4, we have that $\Phi$ is a normalised tight $G$-frame if and only if its Gramian is a $G$-matrix which is a projection. To understand such projections, we now consider the structure of the algebra of $G$-matrices.

Definition. Given a function $\nu: G \rightarrow \mathbb{C}$ let $M(\nu)$ be the $G$-matrix

$$
M(\nu):=\left[\nu\left(g^{-1} h\right)\right]_{g, h \in G} .
$$

Recall that $\mathbb{C} G$, the group algebra of $G$, is the algebra obtained from the complex vector space with basis the elements of $G$, and the multiplication given by extending the multiplication in $G$ linearly.

Proposition 4.7. Let $G$ be a finite group. The sum and product of $G$-matrices is a $G$-matrix. The algebra of $G$-matrices is isomorphic to the group algebra $\mathbb{C} G$ via the map

$$
\pi: M(\nu) \mapsto \sum_{g \in G} \nu(g) g
$$

Proof: $\quad$ Clearly, $M$ is an injective linear map from $\mathbb{C}^{G}$ onto the $G$-matrices, which therefore form a vector space. Moreover, the product of $G$-matrices is a $G$-matrix since

$$
M(\nu) M(\mu)=M(\eta), \quad \eta(g):=\sum_{h \in G} \nu(g h) \mu\left(h^{-1}\right)
$$

so the $G$-matrices form an algebra.

Let $e_{g}: G \rightarrow \mathbb{C}, g \in G$ be the standard basis vectors for $\mathbb{C}^{G}$, given by $e_{g}(h)=\delta_{g, h}$. In view of the natural vector space isomorphism between $\mathbb{C}^{G}$ and $\mathbb{C} G$, it follows that

$$
g \mapsto M\left(e_{g}\right), \quad g \in G
$$

gives a vector space isomorphism between $\mathbb{C} G$ and the $G$-matrices. Further, this is an isomorphism of algebras since $M\left(e_{g_{1}}\right) M\left(e_{g_{2}}\right)=M(\eta)$ where

$$
\eta(g):=\sum_{h \in G} e_{g_{1}}(g h) e_{g_{2}}\left(h^{-1}\right)=e_{g_{1}}\left(g g_{2}^{-1}\right)=e_{g_{1} g_{2}}(g) \quad \Longrightarrow \quad \eta=e_{g_{1} g_{2}} .
$$

Finally, we observe that the inverse of this isomorphism given by (4.9) is (4.8). 
Definition. Let $\rho: G \rightarrow \mathcal{U}(\mathcal{H})$ be a representation of a finite group $G$. The character of $\rho$ is the map $\chi=\chi_{\rho}: G \rightarrow \mathbb{C}$ defined by

$$
\chi(g):=\operatorname{trace}(\rho(g)) .
$$

A character is said to be irreducible if the corresponding representation is irreducible, i.e., $\mathcal{H}$ has no proper nonzero $G$-invariant subspaces. Every character may be uniquely written as a sum of irreducible characters. The irreducible characters $\chi_{1}, \ldots, \chi_{r}$ of $G$ satisfy the orthogonality condition

$$
\frac{1}{|G|} \sum_{g \in G} \chi_{i}(g) \overline{\chi_{j}(g)}=\delta_{i j} .
$$

This leads to a basis of (orthogonal) idempotents $z_{1}, \ldots, z_{r}$ for the centre

$$
Z(\mathbb{C} G):=\{z \in \mathbb{C} G: a z=z a, \forall a \in \mathbb{C} G\}
$$

of $\mathbb{C} G$.

Theorem 4.11. Let $\chi_{1}, \ldots, \chi_{r}$ be the irreducible characters of a finite group $G$, and

$$
z_{i}:=\frac{\chi_{i}(1)}{|G|} \sum_{g \in G} \overline{\chi_{i}}(g) g .
$$

Then $\left\{z_{1}, \ldots, z_{r}\right\}$ is basis for $Z(\mathbb{C} G)$, which satisfies

$$
z_{i}^{2}=z_{i}, \quad z_{i} z_{j}=0, \quad i \neq j .
$$

Proof: This is a standard fact about the group algebra, see, e.g., [JL93:14.714.10].

\section{The classification of central tight $G-$ frames}

A function $\nu: G \rightarrow \mathbb{C}$ is a class function if it is constant on the conjugacy classes of $G$. The irreducible characters form a basis for the space of class functions on $G$, and $\nu$ is a class function if and only if

$$
\sum_{g \in G} \nu(g) g \in Z(\mathbb{C} G)
$$

Definition. A $G$-frame $\Phi=\left(\phi_{g}\right)_{g \in G}$ is said to be central if $\nu: G \rightarrow \mathbb{C}$ defined by

$$
\nu(g):=\left\langle\phi_{1}, \phi_{g}\right\rangle=\left\langle\phi_{1}, g \phi_{1}\right\rangle
$$

is a class function.

For $G$ abelian, all $G$-frames are central (since the conjugacy classes are singletons). In view of Proposition 4.8 and (5.1), a $G$-frame $\Phi$ is central if and only if $\operatorname{Gram}(\Phi)$ is in the centre of the algebra of group matrices. It is easy to see that being central is also equivalent to the 'symmetry condition'

$$
\langle g \phi, h \phi\rangle=\langle g \psi, h \psi\rangle, \quad \forall g, h \in G, \forall \phi, \psi \in \Phi .
$$

We now characterise all central (normalised) tight $G$-frames in terms of the Gramian. 
Theorem 5.2. Let $G$ be a finite group with irreducible characters $\chi_{1}, \ldots, \chi_{r}$. Then $\Phi=\left(\phi_{g}\right)_{g \in G}$ is a central normalised tight $G$-frame if and only if its Gramian is given by

$$
\operatorname{Gram}(\Phi)=M\left(\sum_{i \in I} \frac{\chi_{i}(1)}{|G|} \overline{\chi_{i}}\right)
$$

for some $I \subset\{1, \ldots, r\}$.

Proof: $\quad$ Suppose $\Phi$ is a central normalised tight $G$-frame. Since $\Phi$ is a $G$-frame, by Theorem 4.4 its Gramian is a $G$-matrix

$$
P:=\operatorname{Gram}(\Phi)=M(\nu), \quad \nu(g):=\left\langle\phi_{1}, g \phi_{1}\right\rangle .
$$

Since $\Phi$ is central the above $\nu: G \rightarrow \mathbb{C}$ is a class function. Further, since $\Phi$ is a normalised tight frame, Theorem 2.5 implies that $P$ is an orthogonal projection, in particular $P$ is an idempotent. By applying the isomorphism $\pi$ of (4.8), we have that

$$
u:=\pi(P)=\sum_{g \in G} \nu(g) g
$$

is an idempotent of the group algebra $\mathbb{C} G$, which is central since $\nu$ is a class function. Since $u$ is in $Z(\mathbb{C} G)$, by Theorem 4.11 , we may write it

$$
u=\sum_{i} \alpha_{i} z_{i}, \quad \alpha_{i} \in \mathbb{C}
$$

Since $u$ is an idempotent, (4.13) gives

$$
u^{2}=\sum_{i} \sum_{j} \alpha_{i} \alpha_{j} z_{i} z_{j}=\sum_{i} \alpha_{i}^{2} z_{i}=u=\sum_{i} \alpha_{i} z_{i} \quad \Longrightarrow \quad \alpha_{i}^{2}=\alpha_{i} \quad \Longrightarrow \quad \alpha_{i} \in\{0,1\}
$$

Let $I:=\left\{i: \alpha_{i}=1\right\}$, so that $u=\sum_{i \in I} z_{i}$, and

$$
\begin{aligned}
P & =\pi^{-1}(u)=\sum_{i \in I} \pi^{-1}\left(z_{i}\right)=\sum_{i \in I} \frac{\chi_{i}(1)}{|G|} \sum_{g \in G} \overline{\chi_{i}}(g) \pi^{-1}(g)=\sum_{i \in I} \frac{\chi_{i}(1)}{|G|} \sum_{g \in G} \overline{\chi_{i}}(g) M\left(e_{g}\right) \\
& =M\left(\sum_{i \in I} \frac{\chi_{i}(1)}{|G|} \sum_{g \in G} \overline{\chi_{i}}(g) e_{g}\right)=M\left(\sum_{i \in I} \frac{\chi_{i}(1)}{|G|} \overline{\chi_{i}}\right)
\end{aligned}
$$

Conversely, suppose that $P=\operatorname{Gram}(\Phi)$ has the form (5.3). Since $P$ is a $G$-matrix, Theorem 4.4 implies that $\Phi$ is a $G$-frame. Further, $P$ is a projection matrix, since (4.13) gives

$$
\left(\pi^{-1}(P)\right)^{2}=\left(\sum_{i \in I} z_{i}\right)^{2}=\sum_{i \in I} z_{i}=\pi^{-1}(P) \quad \Longrightarrow \quad P^{2}=P
$$


and $\chi_{i}(1) \in \mathbb{R}$ and $\overline{\chi_{i}}(g)=\chi_{i}\left(g^{-1}\right)$ give

$$
\overline{P_{g, h}}=\sum_{i \in I} \frac{\chi_{i}(1)}{|G|} \chi_{i}\left(g^{-1} h\right)=\sum_{i \in I} \frac{\chi_{i}(1)}{|G|} \overline{\chi_{i}}\left(h^{-1} g\right)=P_{h, g}
$$

Thus it follows from Theorem 2.5 that $\Phi$ is a normalised tight frame. Finally, $\Phi$ is central, since $\chi_{i}(g)=\chi_{i}\left(h^{-1} g h\right)$ implies

$$
\left\langle\phi_{1}, g \phi_{1}\right\rangle=P_{1, g}=\sum_{i \in I} \frac{\chi_{i}(1)}{|G|} \overline{\chi_{i}}(g)=\sum_{i \in I} \frac{\chi_{i}(1)}{|G|} \overline{\chi_{i}}\left(h^{-1} g h\right)=P_{1, h^{-1} g h}=\left\langle\phi_{1}, h^{-1} g h \phi_{1}\right\rangle .
$$

Remark. The condition which ensures $\sum_{g \in G} c_{g} g \in \mathbb{C} G$ corresponds to a self adjoint $G$-matrix under the isomorphism (4.8) is that $\overline{c_{g^{-1}}}=c_{g}, \forall g \in G$.

Independently, [H07:Th.10] has extended the classification of $G$-frames for $G$ abelian given in [VW05:Th.5.4] by using the decomposition of the right regular representation of the group $G$ into $G$-invariant subspaces. In that classification, for a fixed space $\mathcal{H}$ either:

(i) There exist no $G$-frames.

(ii) There exist finitely many $G$-frames.

(iii) There exists infinitely many $G$-frames.

The central normalised tight $G$-frames correspond to the case when there are only finitely many $G$-frames up to equivalence, and give all such frames. The geometric characterisation of such frames being central and their description in terms of characters which follows is not given in [H07].

Corollary 5.4. Let $G$ be a finite group with irreducible characters $\chi_{1}, \ldots, \chi_{r}$. Choose normalised tight $G$-frames $\Phi_{i}$ for $\mathcal{H}_{i}, i=1, \ldots, r$, with

$$
\operatorname{Gram}\left(\Phi_{i}\right)=\frac{\chi_{i}(1)}{|G|} M\left(\overline{\chi_{i}}\right), \quad \operatorname{dim}\left(\mathcal{H}_{i}\right)=\chi_{i}(1)^{2}
$$

e.g., take the columns of $\operatorname{Gram}\left(\Phi_{i}\right)$. Then the unique (up to unitary equivalence) central normalised tight $G$-frame with Gramian (5.3) is given by the direct sum

$$
\bigoplus_{i \in I} \Phi_{i} \subset \mathcal{H}:=\bigoplus_{i \in I} \mathcal{H}_{i}
$$

Further, if $\rho_{i}: G \rightarrow U\left(\mathbb{C}^{d_{i}}\right)$ is a representation with character $\chi_{i}$, then $\Phi_{i}$ can be given as

$$
\Phi_{i}:=\sqrt{\frac{\chi_{i}(1)}{|G|}}\left(\rho_{i}(g)\right)_{g \in G} \subset U\left(\mathbb{C}^{d_{i}}\right) \subset \mathbb{C}^{d_{i} \times d_{i}} \approx \mathbb{C}^{d_{i}^{2}},
$$

where the inner product on the space of $d_{i} \times d_{i}$ matrices is given by $\langle A, B\rangle:=\operatorname{trace}\left(B^{*} A\right)$.

Proof: $\quad$ Applying the isomorphism $\pi$ of Proposition 4.7 gives

$$
\pi\left(\operatorname{Gram}\left(\Phi_{i}\right)\right)=\frac{\chi_{i}(1)}{|G|} \sum_{g \in G} \overline{\chi_{i}}(g) g=z_{i}
$$


where $z_{i}$ is given by (4.12), and so by (4.13)

$$
\operatorname{Gram}\left(\Phi_{i}\right) \operatorname{Gram}\left(\Phi_{j}\right)=\pi^{-1}\left(z_{i}\right) \pi^{-1}\left(z_{j}\right)=\pi^{-1}\left(z_{i} z_{j}\right)=\pi^{-1}(0)=0, \quad i \neq j .
$$

Thus it follows from Corollary 2.7 that the direct sum $\oplus_{i \in I} \Phi_{i}$ is well defined, and its Gramian is given by the formula (5.3). The dimension of $\mathcal{H}_{i}=\operatorname{span} \Phi_{i}$ can be calculated from $(2.6)$

$$
\operatorname{dim}\left(\mathcal{H}_{i}\right)=\operatorname{trace}\left(\operatorname{Gram}\left(\Phi_{i}\right)\right)=\frac{\chi_{i}(1)}{|G|} \sum_{g \in G} \overline{\chi_{i}}(1)=\chi_{i}(1)^{2} .
$$

Finally, if $\Phi_{i}$ is given by (5.6), then we verify

$$
\begin{aligned}
\operatorname{Gram}\left(\Phi_{i}\right)_{g, h} & =\frac{\chi_{i}(1)}{|G|}\left\langle\rho_{i}(g), \rho_{i}(h)\right\rangle=\frac{\chi_{i}(1)}{|G|} \operatorname{trace}\left(\rho_{i}(h)^{*} \rho_{i}(g)\right)=\frac{\chi_{i}(1)}{|G|} \operatorname{trace}\left(\rho_{i}\left(h^{-1} g\right)\right) \\
& =\frac{\chi_{i}(1)}{|G|} \chi_{i}\left(h^{-1} g\right)=\frac{\chi_{i}(1)}{|G|} \overline{\chi_{i}}\left(g^{-1} h\right)=\frac{\chi_{i}(1)}{|G|} M\left(\overline{\chi_{i}}\right)_{g, h},
\end{aligned}
$$

so that $\Phi_{i}$ is indeed a normalised tight $G$-frame with Gramian given by (5.5).

Example 1. Let $\chi_{1}, \ldots, \chi_{s}$ be the 1-dimensional irreducible characters of $G$, so $\chi_{i}(1)=1$. Then Corollary 5.4 gives central tight normalised $G$-frames

$$
\oplus_{i \in I} \Phi_{i}=\oplus_{i \in I} \frac{1}{\sqrt{|G|}}\left(\chi_{i}(g)\right)_{g \in G} \approx \frac{1}{\sqrt{|G|}}\left(\chi_{i}(g)\right)_{i \in I, g \in G}, \quad I \subset\{1, \ldots, s\} .
$$

These can be thought of as being given by (up to a scalar multiplier) the columns of the matrix obtained from the character table $\left(\chi_{i}(g)\right)_{1 \leq i \leq r, g \in G}$ of $G$ by deleting all rows with indices not in the set $I$. In particular, for $G$ abelian, all characters are 1-dimensional, and this gives the result of [VW05:Th.5.4] that in this case all tight $G$-frames (which are central since $G$ is abelian) can be obtained from the character table of $G$ in this way.

Example 2. Let $G=D_{3} \approx S_{3}$ be the dihedral group (symmetric group) of order 6 as given by (4.2), and write class functions and $G$-matrices with respect to the order $1, a, a^{2}, b, a b, a^{2} b$. The conjugacy classes of $G$ are $\{1\},\left\{a, a^{2}\right\},\left\{b, a b, a^{2} b\right\}$, and there are three irreducible characters

$$
\chi_{1}=\left[\begin{array}{l}
1 \\
1 \\
1 \\
1 \\
1 \\
1
\end{array}\right], \quad \chi_{2}=\left[\begin{array}{c}
1 \\
1 \\
1 \\
-1 \\
-1 \\
-1
\end{array}\right], \quad \chi_{3}=\left[\begin{array}{c}
2 \\
-1 \\
-1 \\
0 \\
0 \\
0
\end{array}\right]
$$

Corresponding to each of these, there is a central normalised tight $G$-frame $\Phi_{i}$ for a space of dimension $\chi_{i}(1)^{2}$, with its Gramian matrix $P_{i}$ given by (5.5). These are

$$
P_{1}=\frac{1}{6}\left[\begin{array}{llllll}
1 & 1 & 1 & 1 & 1 & 1 \\
1 & 1 & 1 & 1 & 1 & 1 \\
1 & 1 & 1 & 1 & 1 & 1 \\
1 & 1 & 1 & 1 & 1 & 1 \\
1 & 1 & 1 & 1 & 1 & 1 \\
1 & 1 & 1 & 1 & 1 & 1
\end{array}\right], \quad P_{2}=\frac{1}{6}\left[\begin{array}{cccccc}
1 & 1 & 1 & -1 & -1 & -1 \\
1 & 1 & 1 & -1 & -1 & -1 \\
1 & 1 & 1 & -1 & -1 & -1 \\
-1 & -1 & -1 & 1 & 1 & 1 \\
-1 & -1 & -1 & 1 & 1 & 1 \\
-1 & -1 & -1 & 1 & 1 & 1
\end{array}\right] \text {, }
$$


and

$$
P_{3}=\frac{1}{3}\left[\begin{array}{cccccc}
2 & -1 & -1 & 0 & 0 & 0 \\
-1 & 2 & -1 & 0 & 0 & 0 \\
-1 & -1 & 2 & 0 & 0 & 0 \\
0 & 0 & 0 & 2 & -1 & -1 \\
0 & 0 & 0 & -1 & 2 & -1 \\
0 & 0 & 0 & -1 & -1 & 2
\end{array}\right]
$$

The $\Phi_{i}$ can be obtained by taking the columns of the $P_{i}$, with the action of $a$ and $b$ given by the $G$-matrices

$$
M\left(e_{a}\right)=\left[\begin{array}{cccccc}
0 & 1 & 0 & 0 & 0 & 0 \\
0 & 0 & 1 & 0 & 0 & 0 \\
1 & 0 & 0 & 0 & 0 & 0 \\
0 & 0 & 0 & 0 & 0 & 1 \\
0 & 0 & 0 & 1 & 0 & 0 \\
0 & 0 & 0 & 0 & 1 & 0
\end{array}\right], \quad M\left(e_{b}\right)=\left[\begin{array}{cccccc}
0 & 0 & 0 & 1 & 0 & 0 \\
0 & 0 & 0 & 0 & 1 & 0 \\
0 & 0 & 0 & 0 & 0 & 1 \\
1 & 0 & 0 & 0 & 0 & 0 \\
0 & 1 & 0 & 0 & 0 & 0 \\
0 & 0 & 1 & 0 & 0 & 0
\end{array}\right]
$$

However, we get simpler formulas from (5.6). Since $\chi_{1}$ and $\chi_{2}$ are 1 -dimensional this gives

$$
\Phi_{1}=\frac{1}{\sqrt{6}}(1,1,1,1,1,1), \quad \Phi_{2}=\frac{1}{\sqrt{6}}(1,1,1,-1,-1,-1)
$$

A representation $\rho: D_{3} \rightarrow U\left(\mathbb{C}^{2}\right) \subset \mathbb{C}^{2 \times 2} \approx \mathbb{C}^{4}$ with $\operatorname{trace}(\rho)=\chi_{3}$ is given by

$$
\begin{gathered}
\rho(1)=\left(\begin{array}{ll}
1 & 0 \\
0 & 1
\end{array}\right) \approx\left[\begin{array}{l}
1 \\
0 \\
0 \\
1
\end{array}\right], \quad \rho(a)=\left(\begin{array}{cc}
\omega & 0 \\
0 & \omega^{2}
\end{array}\right) \approx\left[\begin{array}{c}
\omega \\
0 \\
0 \\
\omega^{2}
\end{array}\right], \quad \rho\left(a^{2}\right)=\left(\begin{array}{cc}
\omega^{2} & 0 \\
0 & \omega
\end{array}\right) \approx\left[\begin{array}{c}
\omega^{2} \\
0 \\
0 \\
\omega
\end{array}\right], \\
\rho(b)=\left(\begin{array}{ll}
0 & 1 \\
1 & 0
\end{array}\right) \approx\left[\begin{array}{l}
0 \\
1 \\
1 \\
0
\end{array}\right], \quad \rho(a b)=\left(\begin{array}{cc}
0 & \omega \\
\omega^{2} & 0
\end{array}\right) \approx\left[\begin{array}{c}
0 \\
\omega \\
\omega^{2} \\
0
\end{array}\right], \quad \rho\left(a^{2} b\right)=\left(\begin{array}{cc}
0 & \omega^{2} \\
\omega & 0
\end{array}\right) \approx\left[\begin{array}{c}
0 \\
\omega^{2} \\
\omega \\
0
\end{array}\right],
\end{gathered}
$$

and so we obtain from $(5.6)$

$$
\Phi_{3}=\frac{1}{\sqrt{3}}\left(\left[\begin{array}{l}
1 \\
0 \\
0 \\
1
\end{array}\right],\left[\begin{array}{l}
\omega \\
0 \\
0 \\
\omega^{2}
\end{array}\right],\left[\begin{array}{l}
\omega^{2} \\
0 \\
0 \\
\omega
\end{array}\right],\left[\begin{array}{l}
0 \\
1 \\
1 \\
0
\end{array}\right],\left[\begin{array}{c}
0 \\
\omega \\
\omega^{2} \\
0
\end{array}\right],\left[\begin{array}{c}
0 \\
\omega^{2} \\
\omega \\
0
\end{array}\right]\right)
$$

Thus there are seven central normalised tight $D_{3}$-frames, namely $\Phi_{1}, \Phi_{2} \subset \mathbb{C}, \quad \Phi_{1} \oplus \Phi_{2} \subset \mathbb{C}^{2}, \quad \Phi_{3} \subset \mathbb{C}^{4}, \quad \Phi_{1} \oplus \Phi_{3}, \Phi_{2} \oplus \Phi_{3} \subset \mathbb{C}^{5}, \quad \Phi_{1} \oplus \Phi_{2} \oplus \Phi_{3} \subset \mathbb{C}^{6}$ 
The last of these, $\Phi_{1} \oplus \Phi_{2} \oplus \Phi_{3}$, is an orthonormal basis (which corresponds to the regular representation of $G$ ). We always have such a central normalised tight $G$-frame since the irreducible characters of a finite group $G$ satisfy

$$
|G|=\sum_{i=1}^{r} \chi_{i}(1)^{2}
$$

Example 3. Consider the $D_{3}$-frames $\Phi=\left(\phi_{g}\right)_{g \in D_{3}}$ discussed in the introduction, where

$$
\phi_{1}:=\left[\begin{array}{c}
\cos \theta \\
\sin \theta
\end{array}\right], \quad 0 \leq \theta \leq \frac{\pi}{6}, \quad \rho(a)=\left(\begin{array}{cc}
-\frac{1}{2} & \frac{\sqrt{3}}{2} \\
-\frac{\sqrt{3}}{2} & -\frac{1}{2}
\end{array}\right), \quad \rho(b)=\left(\begin{array}{cc}
1 & 0 \\
0 & -1
\end{array}\right)
$$

For these $\operatorname{Gram}(\Phi)=M(\nu)$, where

$$
\nu=\left[\begin{array}{c}
1 \\
-\frac{1}{2} \\
-\frac{1}{2} \\
2 c^{2}-1 \\
-\sqrt{3} c s-c^{2}+\frac{1}{2} \\
\sqrt{3} c s-c^{2}+\frac{1}{2}
\end{array}\right] \begin{gathered}
1 \\
a \\
a^{2} \\
b \\
a b \\
a^{2} b
\end{gathered}
$$

This cannot be a class function, since by Example 2, the only central tight $D_{3}$-frame for $\mathbb{C}^{2}$ consists of just two vectors (each repeated three times). Since the conjugacy classes of $D_{3}$ are $\{1\},\left\{a, a^{2}\right\},\left\{b, a b, a^{2} b\right\}$, it follows that $\nu$ is a class function if and only if its last three entries are equal. We can try and make $\nu$ close to being a class function by choosing two of these three entries to be equal, e.g.,

$$
\begin{gathered}
\nu(b)=\nu\left(a^{2} b\right) \quad \Longrightarrow \quad 2 c^{2}-1=\sqrt{3} c s-c^{2}+\frac{1}{2} \quad \Longrightarrow \quad \theta=\frac{\pi}{6}, \\
\nu(a b)=\nu\left(a^{2} b\right) \quad \Longrightarrow \quad-\sqrt{3} c s-c^{2}+\frac{1}{2}=\sqrt{3} c s-c^{2}+\frac{1}{2} \quad \Longrightarrow \quad \theta=0,
\end{gathered}
$$

which gives the second and third $D_{3}$-frames of Figure 1. One might argue that these are the most symmetric. An alternative way to try and capture this distance from being a central tight $G$-frame is to consider the length of the error in the projection of $\nu$ onto the class functions $\operatorname{span}\left\{\chi_{i}\right\}_{i=1}^{r}$. In this case, with the characters given by (5.7), we have

$$
E:=\nu-\frac{1}{|G|} \sum_{i=1}^{r}\left\langle\nu, \chi_{i}\right\rangle \chi_{i}=\nu-\frac{1}{2} \chi_{3}=\left[\begin{array}{c}
0 \\
0 \\
0 \\
2 c^{2}-1 \\
-\sqrt{3} c s-c^{2}+\frac{1}{2} \\
\sqrt{3} c s-c^{2}+\frac{1}{2}
\end{array}\right], \quad\|E\|^{2}=\frac{3}{2}
$$


so all the $D_{3}$-frames would be considered equally close to being central. Thus it seems that trying to introduce as many zeros to $E$ as possible rather than minimising its norm may be the best strategy for constructing "nice" frames.

Example 4. Given a central (normalised) tight $G$-frame $\Phi$, one might want to know what are the characters $\left\{\chi_{i}\right\}_{i \in I}$ that it corresponds to. Since $\operatorname{Gram}(\Phi)=M(\nu)$ where

$$
\nu=\sum_{j \in I} \frac{\chi_{j}(1)}{|G|} \overline{\chi_{j}}=1-\text { row of } \operatorname{Gram}(\Phi)=\left(\left\langle\phi_{1}, \phi_{g}\right\rangle\right)_{g \in G}
$$

we have

$$
\sum_{g \in G}\left\langle\phi_{1}, \phi_{g}\right\rangle \chi_{i}(g)=\sum_{g \in G} \sum_{j \in I} \frac{\chi_{j}(1)}{|G|} \overline{\chi_{j}}(g) \chi_{i}(g)=\sum_{j \in I} \frac{\chi_{j}(1)}{|G|}\left\langle\chi_{i}, \chi_{j}\right\rangle .
$$

Hence from the orthogonality (4.10) of the irreducible characters it follows that

$$
I=\left\{i: \sum_{g \in G}\left\langle\phi_{1}, \phi_{g}\right\rangle \chi_{i}(g) \neq 0\right\}
$$

For example, let $C_{3}=\langle a\rangle$ be the cyclic group of order 3 with $a$ acting as rotation by $\frac{2 \pi}{3}$, and $v$ be a unit vector in $\mathbb{R}^{2}$. Then $\Phi=\left(\phi_{1}, \phi_{a}, \phi_{a^{2}}\right)=\left(v, a v, a^{2} v\right)$ is central tight $C_{3}$-frame. Let $\chi_{1}=(1,1,1), \chi_{2}=\left(1, \omega, \omega^{2}\right), \chi_{3}=\left(1, \omega^{2}, \omega\right)$ be the characters of $C_{3}$ given by the character table (1.1). We calculate

$$
\sum_{g \in C_{3}}\left\langle\phi_{1}, \phi_{g}\right\rangle \chi_{1}(g)=1-\frac{1}{2}-\frac{1}{2}=0, \quad \sum_{g \in C_{3}}\left\langle\phi_{1}, \phi_{g}\right\rangle \chi_{i}(g)=1-\frac{1}{2} \omega-\frac{1}{2} \omega^{2}=\frac{3}{2}, \quad i=2,3,
$$

and hence $\Phi$ is obtained by deleting the first row of the character table.

\section{Conclusion}

We have shown that tight frames which occur as the orbit of a single vector under a unitary action of a finite group $G$ (normalised tight $G$-frames) can be identified with elements $p$ of the group algebra $\mathbb{C} G$ which satisfy

$$
p^{2}=p, \quad \bar{p}=p
$$

where

$$
\bar{p}:=\sum_{g \in G} \overline{c_{g}} g^{-1}=\sum_{g \in G} \overline{c_{g^{-1}}} g, \quad p=\sum_{g \in G} c_{g} g \in \mathbb{C} G .
$$

We then singled out a finite subset of the so called central normalised tight $G$-frames for which $p$ belongs to the centre of $\mathbb{C} G$, and gave a complete decription of these in terms of the irreducible characters of $G$. We hope that this new technique of describing $G$-frames in terms of the group algebra of $G$ will be used to analyse and construct optimal $G$-frames for various applications. 


\section{References}

[AMS05] J. T. Astola, C. Moraga, and R. S. Stanković, "Fourier Analysis on Finite Groups with Applications in Signal Processing and System Design", Wiley, New Jersey, 2005.

[BK00] R. Bernardini and J. Kovačević, Designing local orthogonal bases on finite groups II: Nonabelian case, J. Fourier Anal. Appl. 6 (2000), 207-231.

[BE03] H. Bölcskei and Y. C. Eldar, Geometrically uniform frames, IEEE Trans. Inform. Theory 49 no. 4 (2003), 993-1006.

[BR04] V. M. Bukhshtaber and E. G. Ris, Rings of continuous functions, symmetric products, and Frobenius algebras, Russian Math. Surveys 59 (2004), 125-145.

[CK07] A. Chebira and J. Kovačević, Life Beyond Bases: The Advent of Frames (Part I), IEEE Signal Processing Magazine (to appear), 2007.

[D79] P. J. Davis, "Circulant matrices", Wiley, New York, 1979.

[EF01] Y. C. Eldar and G. D. Forney, On quantum detection and the square-root measurement, IEEE Trans. Inform. Theory 47 (2001), 858-872.

[GH04 ${ }_{1}$ J.-P. Gabardo and D. Han, The uniqueness of the dual of Weyl-Heisenberg subspace frames, Appl. Comput. Harmonic Anal. 17 (2004), 226-240.

[GH04 2 J.-P. Gabardo and D. Han, Balian-Low phenomenon for subspace Gabor frames, $J$. Math. Phys. 45 (2004), 3362-3378.

[HL00] D. Han and D. R. Larson, Frames, bases and group representations, Mem. Amer. Math. Soc. 147 no. 697 (2000), 94 pp.

[H07] D. Han, Classification of Finite Group-Frames and Super-Frames, Canad. Math. Bull. 50 (2007), 85-96.

[I06] I. M. Isaacs, "Character Theory of Finite Groups. Corrected reprint of the 1976 original", AMS Chelsea, Providence, 2006.

[JL93] G. James and M. Liebeck, "Representations and Characters of Groups", Cambridge University Press, Cambridge, 1993.

[J07] K. W. Johnson, "Group representation theory via group matrices and group determinants", Book, in preparation, 2007.

[L77] W. Ledermann, "Introduction to Group Characters", Cambridge University Press, Cambridge, 1977.

[RW02] R. Reams and S. Waldron, Isometric tight frames, Electron. J. Linear Algebra 9 (2002), 122-128.

[VW05] R. Vale and S. Waldron, Tight frames and their symmetries, Constr. Approx. 21 (2005), 83-112. 